OPEN ACCESS

Edited by:

Vladimir Litvak,

UCL Institute of Neurology, UK

Reviewed by: Joseph Classen, Leipzig University, Germany

Daniele Belvisi, Istituto Neurologico Mediterraneo Neuromed (IRCCS), Italy

${ }^{*}$ Correspondence: Anastasia Shulga anastasia.shulga@helsinki.fi

Received: 22 June 2016 Accepted: 06 September 2016 Published: 23 September 2016

Citation:

Shulga A, Zubareva A, Lioumis P and Mäkelä JP (2016) Paired Associative Stimulation with High-Frequency Peripheral Component Leads to Enhancement of Corticospinal Transmission at Wide Range of Interstimulus Intervals.

Front. Hum. Neurosci. 10:470. doi: 10.3389/fnhum.2016.00470

\section{Paired Associative Stimulation with High-Frequency Peripheral Component Leads to Enhancement of Corticospinal Transmission at Wide Range of Interstimulus Intervals}

\author{
Anastasia Shulga $^{1,2 *}$, Aleksandra Zubareva ${ }^{1}$, Pantelis Lioumis ${ }^{1}$ and Jyrki P. Mäkelä ${ }^{1}$ \\ ' BioMag Laboratory, HUS Medical Imaging Center, University of Helsinki and Helsinki University Hospital, Helsinki, Finland, \\ ${ }^{2}$ Clinical Neurosciences, Neurology, University of Helsinki and Helsinki University Hospital, Helsinki, Finland
}

Background: In spinal paired associative stimulation (PAS), orthodromic and antidromic volleys elicited by transcranial magnetic stimulation (TMS) and peripheral nerve stimulation (PNS) coincide at corticomotoneuronal synapses at the spinal cord. The interstimulus interval (ISI) between TMS and PNS determines whether PAS leads to motor-evoked potential (MEP) potentiation or depression. PAS applied as a long-term treatment for neurological patients might alter conduction of neural fibers over time. Moreover, measurements of motoneuron conductance for determination of ISIs may be challenging in these patients.

Results: We sought to design a PAS protocol to induce MEP potentiation at wide range of ISIs. We tested PAS consisting of high-intensity (100\% stimulator output, SO) TMS and high-frequency $(50 \mathrm{~Hz})$ PNS in five subjects at five different ISIs. Our protocol induced potentiation of MEP amplitudes in all subjects at all tested intervals. TMS and PNS alone did not result in MEP potentiation. The variant of PAS protocol described here does not require exact adjustment of ISIs in order to achieve effective potentiation of MEPs.

Conclusions: This variant of PAS might be feasible as a long-term treatment in rehabilitation of neurological patients.

\footnotetext{
Keywords: paired associative stimulation, transcranial magnetic stimulation, plasticity, spinal cord, peripheral electrical stimulation
}

\section{INTRODUCTION}

Paired associative stimulation (PAS) is a technique where transcranial magnetic stimulation (TMS) is synchronized with peripheral nerve electrical stimulation (PNS); signals are timed to coincide at synapses at cortical (Stefan et al., 2000) level to enhance corticospinal excitability. In spinal PAS, signals are timed to coincide at the spinal cord level (Taylor and Martin, 2009; Cortes et al., 2011; Leukel et al., 2012; Shulga et al., 2015). PAS protocol typically consists of a single TMS pulse combined either with a single PNS pulse or 10-Hz PNS trains (Carson and Kennedy, 2013).

These conventional protocols lead to long-term potentiation (LTP)-like effect at a limited range of interstimulus interval (ISIs; Bunday and Perez, 2012; Carson and Kennedy, 2013; Shulga et al., 2015). If PAS would be applied in multiple sessions to enhance corticospinal 
connections as a tool for long-term rehabilitation, the initially calculated ISI would need to be adjusted constantly, as neuronal conductivity may change over time during recovery of the injury. Moreover, it is plausible that the remaining neural pathways in patients with neurological diseases have a wide range of conductivities as a result of partial injuries; precise measurements of conductivities in such patients can be challenging.

Whereas PAS protocols with single peripheral pulses are best suited for studying plasticity mechanisms, more clinically feasible types of PAS protocols are required for medical treatment development. Such protocol should reliably enhance corticospinal transmission at a wide range of ISIs. One possibility to design such protocol is to increase the number of interactions between pre- and postsynaptic volleys through the increase of volley number: when LTP-inducing and LTD-inducing timing interactions occur at the same time, LTP can override LTD (Sjöström et al., 2001). The increase in the number of orthodromic volleys can be achieved by increasing TMS intensity; high-intensity TMS pulses result in a high-frequency repetitive discharge of corticospinal neurons (Di Lazzaro et al., 2008). To increase the number of antidromic volleys, highfrequency trains of PNS can be used.

The potential of single-session spinal PAS as rehabilitation for spinal cord injury patients, and of multiple-session cortical PAS for stroke patients has been reported (Uy et al., 2003; Bunday and Perez, 2012). We have recently published a case report of two incomplete spinal cord injury patients showing the positive long-term effect of multiple-session spinal PAS consisting of single high-intensity TMS pulses combined with $50 \mathrm{~Hz}$ PNS trains (Shulga et al., 2016). Here we characterize single-session PAS with these parameters in healthy subjects. We show that this PAS protocol reliably leads to potentiation of motor-evoked potential (MEP) amplitudes at a wide range of ISIs.

\section{MATERIALS AND METHODS}

\section{Subjects}

Five subjects (3 males; right-handed; age 30-60, mean age 40) participated in the study. Each subject participated in 10 experiments, conducted on different days. The study was conducted as a part of clinical trial approved by Helsinki University Central Hospital medical ethical committee; a written informed consent was obtained from each subject.

\section{Determination of Interstimulus Interval}

We determined ISIs $0 \mathrm{~ms}$ (antidromic and orthodromic pulses arriving simultaneously at corticomotoneuronal synapses at the spinal cord level) between TMS pulse and the first pulse of the peripheral train with the formula $\mathrm{F}$ latency minus MEP latency, as described previously (Shulga et al., 2015). We used minimal F-latency (the shortest latency in a series of 10 measurements) at supramaximal stimulation (the stimulation intensity at which increasing the intensity does not further produce the increase in $\mathrm{F}$ wave amplitude) with $0.2 \mathrm{~ms}$ pulses. MEPs were elicited by TMS at $120 \%$ of resting motor threshold (RMT). RMT was determined as the minimum intensity required to produce a MEP over $50 \mu \mathrm{V}$ in over $50 \%$ times in a series of 10 pulses.

\section{Navigated Transcranial Magnetic Stimulation}

TMS was given with eXimia magnetic stimulator (Nexstim Ltd, Helsinki, Finland). For stimulation during PAS session, TMS was given at 100\% SO; for measurement of MEPs, TMS was given at $120 \%$ RMT (see above). We used figure-of eight coil; the outer diameter of the coil was $70 \mathrm{~mm}$. The induced current was oriented perpendicular to the sulcus at the stimulation target at the beginning of the mapping. The optimal site for TMS was determined individually for each subject based on the mapping of the motor cortex: the spot and coil orientation most readily eliciting MEPs from abductor hallucis muscle was selected. The selected spots and coil orientation were registered in our MRI-guided navigation system (NBS navigation system, Nexstim Ltd, Helsinki, Finland). The navigation system ensured that the TMS stimulation target and coil orientation of the PAS protocol was the same as the stimulation site used for MEP measurements.

\section{Peripheral Electrical Stimulation}

We delivered PNS using Dantec Keypoint ${ }^{\circledR}$ electroneuromyography device (Natus Medical Incorporated, Pleasanton, CA, USA). Peripheral electric stimulation was delivered as $50 \mathrm{~Hz}$ trains of 1-ms square wave pulses for $100 \mathrm{~ms}$ (= 6 pulses per train) to depolarize lower motor neurons' somata and dendrites by antidromic motor neuron volleys. The individual minimum intensity required to produce F-responses when measured with single 1 -ms pulses was used for the $50-\mathrm{Hz}$ trains.

Two subjects (2 and 3) perceived peripheral stimulation as unpleasant. Their skin was locally anesthetized prior to the experiments with $2.5 \%$ lidocaine/prilocaine ointment (EMLA $\left.{ }^{\circledR}\right)$. EMLA penetrates 3-5 mm into the skin (Gajraj et al., 1994) and thus does not affect the conductivity of the tibial nerve. Parameters used for each subject are presented in Table 1.

\section{Paired Associative Stimulation}

Both peripheral stimulation and TMS were triggered by Presentation ${ }^{\circledR}$ software (Neurobehavioral Systems Inc., Albany, NY, USA) to guarantee the adjusted ISIs. The peripheral electric stimulation train was delivered once every $5 \mathrm{~s}$, each train synchronized with single-pulse TMS, for $20 \mathrm{~min}$. All experiments were applied to left motor cortex/right tibial nerve.

During the stimulations (PAS or PNS only), subjects were instructed to mentally concentrate on the movement produced by electrical stimulation of the tibial nerve (toes flexion of the right leg), but not to attempt to move the leg or toes during the stimulation.

\section{Measurements of MEPs}

Ten MEPs were measured at 120\% RMT, as described above, immediately before and after the stimulation. For graphical presentation of the results (Figure 1), the MEP traces were 


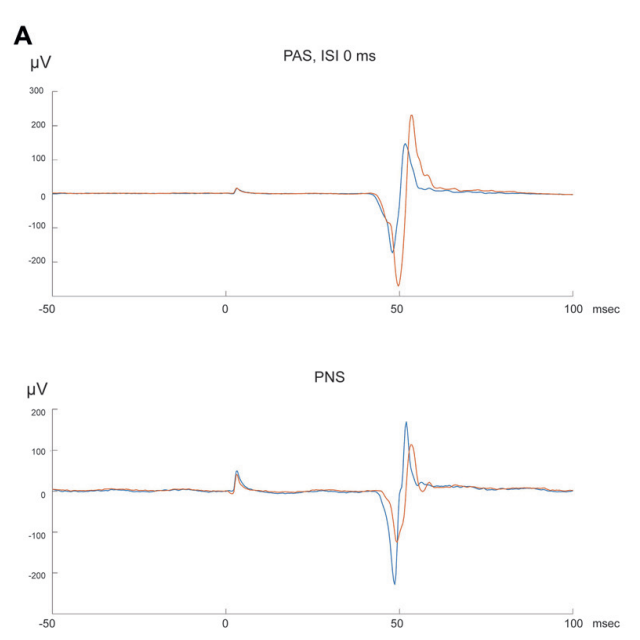

B

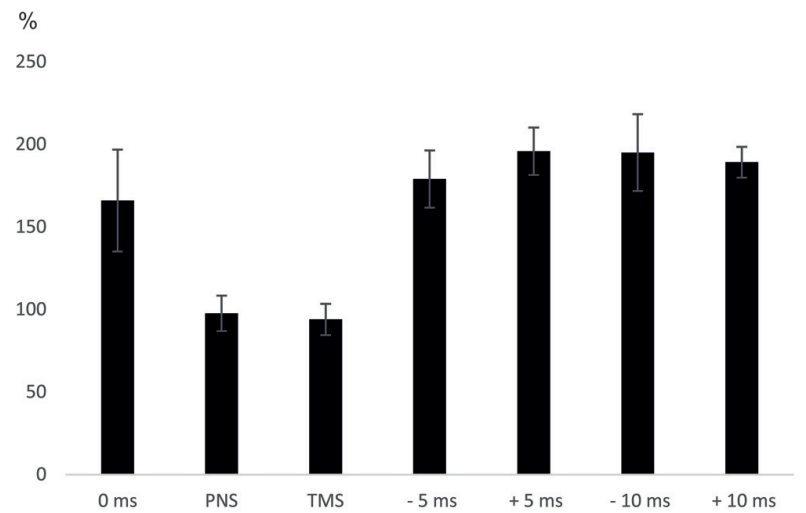

FIGURE 1 | (A) An average of 10 motor-evoked potentials (MEPs) recorded in a representative subject (subject 2). Blue-pre-paired associative stimulation (PAS)/peripheral nerve stimulation (PNS), red-post-PAS/PNS. (B) Summary of the results.

averaged using MATLAB (MathWorks Ltd., Nattick, MA, USA) software.

\section{Statistics}

To compare the averages of MEP amplitudes pre-PAS vs. postPAS, we used Wilcoxon signed ranks test. To investigate whether there is a difference between PAS groups with different ISIs, we used Kruskal-Wallis test. Statistical tests were done on IBM SPSS Statistics 22 Software. Data is presented as mean \pm standard error.

\section{RESULTS}

Each subject received PAS sessions at intervals $0 \mathrm{~ms}, \pm 5 \mathrm{~ms}$ and $\pm 10 \mathrm{~ms}$ as well as $300 \mathrm{~ms}$, one PNS only, and one TMS only session. The detailed results are presented in Table 2.

After PAS with $0 \mathrm{~ms}$ ISI, MEP amplitudes increased to $166 \pm 31 \%$ ( $p=0.043$ post vs. pre- PAS), whereas after PNS only, there was no change in MEP amplitudes ( $98 \pm 11 \%$ post vs. pre-PNS, $p=0.69)$. TMS only did not induce MEP amplitude potentiation either ( $94 \pm 10 \%$ post vs. pre-TMS, $p=0.89$ ). MEP latencies did not change after PAS (post-PAS minus pre-PAS: $0.1 \pm 0.4 \mathrm{~ms}, p=0.69$ ) and after TMS (post-TMS minus preTMS: $0.88 \pm 0.82 \mathrm{~ms}, p=0.08)$ and became slightly prolonged after PNS (post-PNS minus pre-PNS: $1.1 \pm 0.3 \mathrm{~ms}, p=0.043$ ). At $30 \mathrm{~min}$ and $60 \mathrm{~min}$ after PAS with $0 \mathrm{~ms}$ ISI, there was a trend towards higher MEP amplitudes ( $184 \pm 54 \%, p=0.08$, at $30 \mathrm{~min}$ and $139 \pm 31 \%, p=0.08$, at $60 \mathrm{~min}$ ); the potentiation persisted at least for $60 \mathrm{~min}$ in four out of five subjects (see Table 2).

PAS increased MEP amplitudes at all tested intervals: $+5 \mathrm{~ms}$ $(196 \pm 14 \%, p=0.043),-5 \mathrm{~ms}(180 \pm 17 \%, p=0.043),+10 \mathrm{~ms}$ $(189 \pm 9 \%, p=0.043)$ and $-10 \mathrm{~ms}(195 \pm 23 \%, p=0.043)$. The MEP latencies did not change (post-PAS minus pre-PAS, +5 ms ISI: $0.6 \pm 0.5, p=0.18 ;-5$ ms ISI: $-0.1 \pm 0.2, p=0.5$; $+10 \mathrm{~ms}:-0.9 \pm 0.7 \mathrm{~ms}, p=0.23 ;-10 \mathrm{~ms}:-0.3 \pm 0.1 \mathrm{~ms}$, $p=0.08)$. Although MEP amplitude increase at $\pm 5 \mathrm{~ms}$ and $\pm 10 \mathrm{~ms}$ ISI was larger than at $0 \mathrm{~ms}$ ISI (Table 2), amplitude increases did not differ significantly between the groups $(p=0.58$ by Kruskal-Wallis test). In order to test an interval where TMS and PNS-induced volleys are not timed to arrive simultaneously, we applied PAS at ISI $300 \mathrm{~ms}$ (that is, TMS coming $200 \mathrm{~ms}$ after the last PNS pulse); at this interval, there was no significant increase in MEP amplitudes $(157 \pm 41 \%, p=0.5)$.

All experiments mentioned above were conducted in the presence of motor imagery (see "Materials and Methods" Section). We conducted a separate experiment without motor imagery at ISI $0 \mathrm{~ms}$; PAS induced MEP potentiation also without motor imagery $(198 \pm 28 \%, p=0.043$, Table 2$)$.

TABLE 1 | Settings used for each subject.

\begin{tabular}{|c|c|c|c|c|c|c|c|c|c|c|}
\hline Subject & $\begin{array}{c}\text { RMT } \\
\text { (\% of SO) }\end{array}$ & $\begin{array}{c}\text { PNS intensity } \\
\text { (mA) }\end{array}$ & EMLA & $\begin{array}{l}\text { F latency } \\
\text { (ms) }\end{array}$ & $\begin{array}{c}\text { MEP latency } \\
\text { (ms) }\end{array}$ & $\begin{array}{l}\text { ISI } 0 \text { ms } \\
\text { (ms) }\end{array}$ & $\begin{array}{c}\text { ISI }-5 \mathrm{~ms} \\
(\mathrm{~ms})\end{array}$ & $\begin{array}{l}\text { ISI }+5 \mathrm{~ms} \\
(\mathrm{~ms})\end{array}$ & $\begin{array}{c}\text { ISI -10 ms } \\
\text { (ms) }\end{array}$ & $\begin{array}{c}\text { ISI }+10 \mathrm{~ms} \\
(\mathrm{~ms})\end{array}$ \\
\hline 1 & 83 & 11 & - & 50 & 42 & 8 & 3 & 13 & -2 & 18 \\
\hline 2 & 52 & 5 & + & 48 & 43 & 5 & 0 & 10 & -5 & 15 \\
\hline 3 & 64 & 9 & + & 58 & 48 & 10 & 5 & 15 & 0 & 20 \\
\hline 4 & 61 & 20 & - & 50 & 48 & 2 & -3 & 7 & -8 & 12 \\
\hline 5 & 61 & 6 & - & 52 & 43 & 9 & 4 & 14 & -1 & 19 \\
\hline
\end{tabular}

SO-stimulator output, Positive ISI-PNS precedes TMS, negative ISI-TMS precedes PNS. 
TABLE 2 | Detailed results shown separately for each subject.

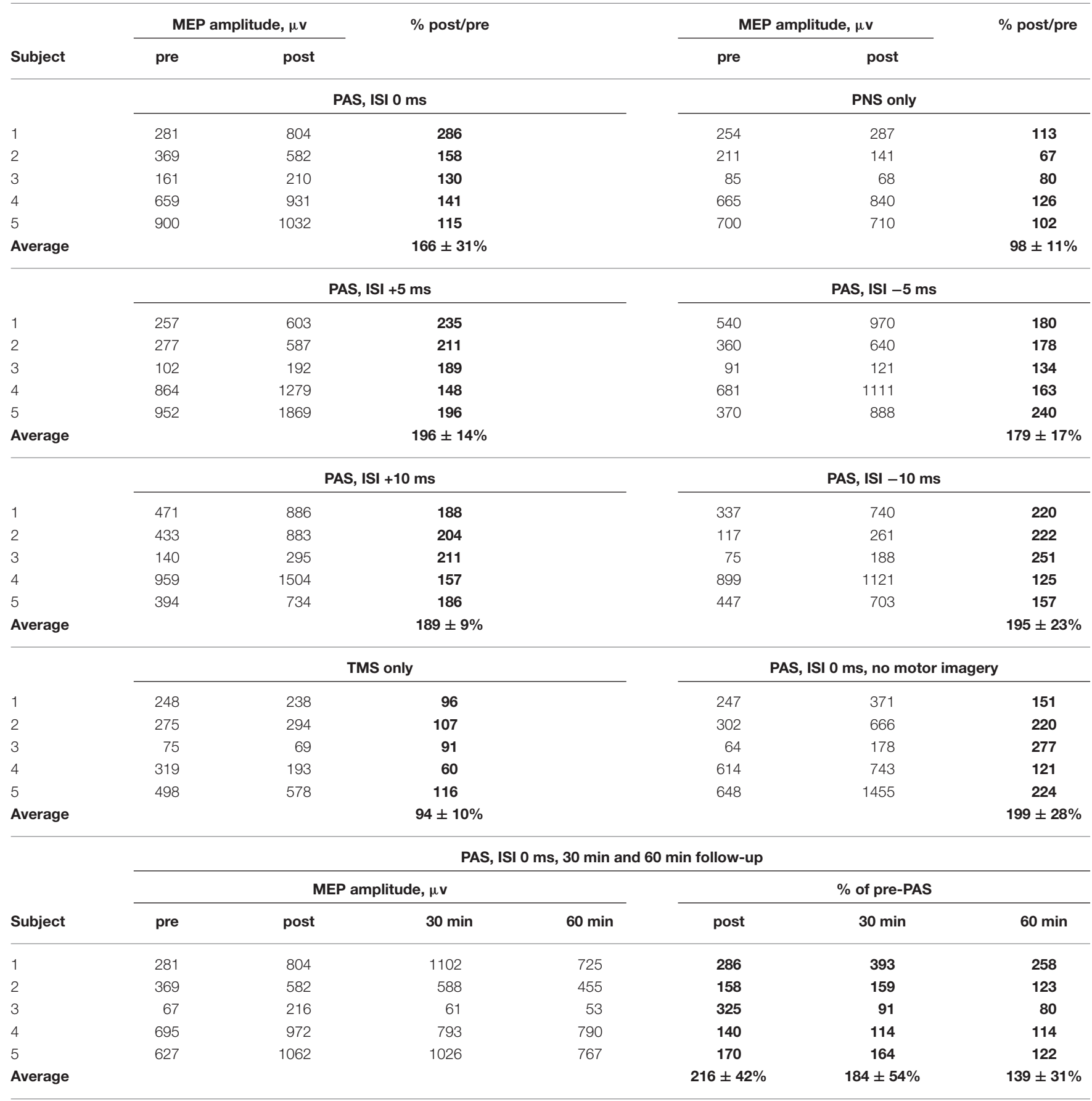

\begin{tabular}{|c|c|c|c|}
\hline \multirow[b]{3}{*}{ Subject } & \multicolumn{3}{|c|}{ PAS, ISI 300 ms } \\
\hline & \multicolumn{2}{|c|}{ MEP amplitude, $\mu v$} & \multirow[t]{2}{*}{$\%$ post/pre } \\
\hline & pre & post & \\
\hline 1 & 271 & 298 & 110 \\
\hline 2 & 322 & 246 & 76 \\
\hline 3 & 94 & 263 & 280 \\
\hline 4 & 1050 & 932 & 88 \\
\hline 5 & 258 & 595 & 230 \\
\hline Average & & & $157 \pm 41 \%$ \\
\hline
\end{tabular}




\section{DISCUSSION}

Our PAS protocol reliably induced MEP potentiation at a wide range of ISIs in a robust way in healthy subjects, and might thus be more suitable for clinical use than conventional PAS protocols. The conventional PAS protocol has a single-pulse TMS and PNS; its properties have been investigated in detail (Carson and Kennedy, 2013). In this work, we wanted to present a new variant of PAS protocol. We have previously examined the properties of another variant of conventional PAS which utilized $10 \mathrm{~Hz}$ PNS (Shulga et al., 2015) with the same equipment and ISI determination as presented here; this protocol did not induce MEP potentiation at $\pm 10 \mathrm{~ms}$ ISI. TMS pulses were given at maximum intensity to ensure multiple collisions of pre- and postsynaptic pulses at the spinal cord level. In neurological patients, upper motor neurons might be less excitable than in healthy subjects, and high-intensity TMS would ensure their excitation.

For PNS, we used high-frequency peripheral pulses at minimal intensity required to elicit F-response to ensure the activation of the motor neurons. We have previously reported the use of otherwise identical protocol in one healthy subject at intensity higher than minimal intensity for F-response $(15 \mathrm{~mA})$; this has led to MEP potentiation observable at $1 \mathrm{~h}$, but not immediately after PAS (Shulga et al., 2016). Lowering the intensity in the same subject to $11 \mathrm{~mA}$ (subject 1 in this study) has led to observable MEP potentiation immediately after the protocol. It is probable that both intensities enhance corticospinal transmission immediately, but higher intensity leads to exhaustion of the muscle which covers the effect.

The ensuing peripheral stimulation was unpleasant for some subjects. The use of EMLA ointment reduced these unpleasant sensations. Gradual adaptation to the peripheral stimulation during subsequent sessions was also reported. In neurological patients, peripheral nerves can be less excitable than in healthy subjects, and even higher stimulation intensities might be required. The possibility to use EMLA as well as the adaptation to the unpleasant properties of the PNS during the sessions should be kept in mind when designing PAS protocols for these patients.

The use of TMS or PNS alone did not lead to potentiation of MEP amplitudes. The control experiment where we applied TMS $300 \mathrm{~ms}$ after first PNS pulse (that is, $200 \mathrm{~ms}$ after the last PNS pulse) resulted at a group level in MEP potentiation within the same range as other tested intervals. The result was, however, highly variable between the subjects as both decreases and increases of MEPs were observed, and not significant (see Table 2). It is plausible that depolarization of lower motor neurons induced by PNS has different duration in different subjects. When TMS and PNS are timed to coincide closely on spinal cord, and possibly also cortical, level, there is a stable MEP amplitude increase in all subjects. However, when PNS and TMS pulses are as far as $200 \mathrm{~ms}$ apart, there is an interaction between antidromic and orthodromic depolarization only in part of the subjects.

The use of high-frequency PNS naturally hampers the interpretation of the neural level of the physiological effects, as the relation between the TMS- and PNS-induced activations gets more complicated. In the protocol presented here, the arrival timing between the pre-and postsynaptic volleys at the spinal cord level is way much closer than possible interactions occurring at the cortical level; it is thus plausible that observed MEP potentiation originates mainly from induction of plasticity at corticomotoneuronal synapses of the spinal cord. However, it is plausible that also somatosensory afferents are activated, and interactions occur at multiple levels. Further research is needed to elucidate the mechanism of MEP amplitude potentiation by the protocol presented here more precisely.

We conducted all experiments in the presence of motor imagery, since we used this method on our spinal cord injury patients (Shulga et al., 2016). Control experiment without motor imagery revealed that it is not required for MEP amplitude potentiation in healthy subjects at $0 \mathrm{~ms}$ ISI. However, this observation should be interpreted with caution when designing the protocols for neurological patients: in these patients, RMTs can be much higher than in healthy subjects, and lowering RMT through motor imagery might be helpful to achieve the desired effect of PAS.

Spike-time dependent plasticity (STDP) is dependent on numerous factors: the firing rate, the number of coactive synaptic inputs, the postsynaptic voltage and the timing of the inputs, among others (Feldman, 2012). Importantly for this protocol, it has been shown in vitro that spike-timing relationships causing LTP can "win" out over those favoring LTD when multiple interactions occur at the same time (Sjöström et al., 2001).

\section{CONCLUSION}

Our aim was to develop and test tools for patient rehabilitation, and the observed increase of efficacy of the motor activation by the PAS is promising in this respect. The results support the usefulness of the stimulation parameters selected for our proof of concept-study of PAS used in rehabilitation of two patients with spinal cord injuries (Shulga et al., 2016).

\section{AUTHOR CONTRIBUTIONS}

AS, AZ, PL and JPM: conception and design of the work, acquisition, analysis, and interpretation of data for the work, drafting the work and revising it critically for important intellectual content, final approval of the version to be published, agreement to be accountable for all aspects of the work.

\section{FUNDING}

This study was supported in part by research grants from the Faculty of Medicine, University of Helsinki, Emil Aaltonen Foundation and Finnish Cultural Foundation.

\section{ACKNOWLEDGMENTS}

We thank our subjects for participation in the study, and Victor Pavlushkov for the help with MATLAB. 


\section{REFERENCES}

Bunday, K. L., and Perez, M. A. (2012). Motor recovery after spinal cord injury enhanced by strengthening corticospinal synaptic transmission. Curr. Biol. 22, 2355-2361. doi: 10.1016/j.cub.2012.10.046

Carson, R. G., and Kennedy, N. C. (2013). Modulation of human corticospinal excitability by paired associative stimulation. Front. Hum. Neurosci. 7:823. doi: 10.3389/fnhum.2013.00823

Cortes, M., Thickbroom, G. W., Valls-Sole, J., Pascual-Leone, A., and Edwards, D. J. (2011). Spinal associative stimulation: a non-invasive stimulation paradigm to modulate spinal excitability. Clin. Neurophysiol. 122, 2254-2259. doi: 10.1016/j.clinph.2011.02.038

Di Lazzaro, V., Ziemann, U., and Lemon, R. N. (2008). State of the art: physiology of transcranial motor cortex stimulation. Brain Stimul. 1, 345-362. doi: 10. 1016/j.brs.2008.07.004

Feldman, D. E. (2012). The spike-timing dependence of plasticity. Neuron 75 , 556-571. doi: 10.1016/j.neuron.2012.08.001

Gajraj, N. M., Pennant, J. H., and Watcha, M. F. (1994). Eutectic mixture of local anesthetics (EMLA) cream. Anesth. Analg. 78, 574-583. doi: 10.1213/00000539199403000-00026

Leukel, C., Taube, W., Beck, S., and Schubert, M. (2012). Pathway-specific plasticity in the human spinal cord. Eur. J. Neurosci. 35, 1622-1629. doi: 10. 1111/j.1460-9568.2012.08067.x

Shulga, A., Lioumis, P., Kirveskari, E., Savolainen, S., Makela, J. P., and Ylinen, A. (2015). The use of F-response in defining interstimulus intervals appropriate for LTP-like plasticity induction in lower limb spinal paired associative stimulation. J. Neurosci. Methods 242, 112-117. doi: 10.1016/j.jneumeth.2015. 01.012
Shulga, A., Lioumis, P., Zubareva, A., Brandstack, N., Kuusela, L., Kirveskari, E., et al. (2016). Long-term paired associative stimulation can restore voluntary control over paralyzed muscles in incomplete chronic spinal cord injury patients. Spinal Cord Ser. Cases 2:16016. doi: 10.1038/scsandc. 2016.16

Sjöström, P. J., Turrigiano, G. G., and Nelson, S. B. (2001). Rate, timing and cooperativity jointly determine cortical synaptic plasticity. Neuron 32, 1149-1164. doi: 10.1016/s0896-6273(01)00542-6

Stefan, K., Kunesch, E., Cohen, L. G., Benecke, R., and Classen, J. (2000). Induction of plasticity in the human motor cortex by paired associative stimulation. Brain 123, 572-584. doi: 10.1093/brain/123.3.572

Taylor, J. L., and Martin, P. G. (2009). Voluntary motor output is altered by spiketiming-dependent changes in the human corticospinal pathway. J. Neurosci. 29, 11708-11716. doi: 10.1523/jneurosci.2217-09.2009

Uy, J., Ridding, M. C., Hillier, S., Thompson, P. D., and Miles, T. S. (2003). Does induction of plastic change in motor cortex improve leg function after stroke? Neurology 61, 982-984. doi: 10.1212/01.wnl.0000078809.33581.1f

Conflict of Interest Statement: PL reports personal fees from Nexstim Ltd, outside the submitted work. Other authors have nothing to disclose.

Copyright (c) 2016 Shulga, Zubareva, Lioumis and Mäkelä. This is an open-access article distributed under the terms of the Creative Commons Attribution License (CC BY). The use, distribution and reproduction in other forums is permitted, provided the original author(s) or licensor are credited and that the original publication in this journal is cited, in accordance with accepted academic practice. No use, distribution or reproduction is permitted which does not comply with these terms. 